\title{
Parameter Identification of Induction Motors using Variable-weighted Cost Function of Genetic Algorithms
}

\author{
A.C. Megherbi ${ }^{\dagger}$, H. Megherbi*, K. Benmahamed $* *$, A.G. Aissaoui*** and A. Tahour***
}

\begin{abstract}
This paper presents a contribution to parameter identification of a non-linear system using a new strategy to improve the genetic algorithm (GA) method. Since cost function plays an important role in GA-based parameter identification, we propose to improve the simple version of GA, where weights of the cost function are not taken as constant values, but varying along the procedure of parameter identification. This modified version of GA is applied to the induction motor (IM) as an example of nonlinear system. The GA cost function is the weighted sum of stator current and rotor speed errors between the plant and the model of induction motor. Simulation results show that the identification method based on improved GA is feasible and gives high precision.
\end{abstract}

Keywords: Genetic algorithm, Induction motor, Parameter identification, Cost function

\section{Introduction}

Approximately $50 \%-60 \%$ of electric energy is consumed by induction motors (IM) in a power system. Therefore, IM are a significant load and are the most widely used motors in the industry. They are easy to build, rugged, reliable, and have a good self-starting capability. Majority of control schemes of such motor drives require exact knowledge of at least some of the induction motor parameters. A mismatch between the actual motor values and values used within the controller leads to deterioration in the drive performance [1]. To overcome these problems, some works have been done towards determining the parameters of induction motor prior and selection of the appropriate models for study [2].

Induction motors are highly nonlinear and electric rotor variables are not measurable. More commonly, electromagnetic parameters are not precisely known. The skin effect in the rotor winding and iron core saturation lead to even bigger complications in the modeling process of the machine. Hence, the source for the determination of such parameters is based usually on indirect measurement methods where the input is a generic, such as input stator voltage or rotational speed of the motor. The output of the model is compared with the measured one.

Conventional optimization techniques have been applied to this type of problem with limited success, although with the inherent problem of convergence to a local minimum instead of a global one. The optimum parameter values determined by these techniques heavily depend on the ini-

$\dagger \quad$ Corresponding Author: Dept. of Electrical Engineering, Med Khider University, Biskra, Algeria. (megherbic@yahoo.fr)

* Dept. of Electrical Engineering, Med Khider University, Biskra, Algeria.

** Dept. of Electronic Engineering, Ferhat Abass University, Setif, Algeria.

*** Dept. of Electrical Engineering, Bechar University, Algeria.

Received: November 4, 2009; Accepted: July 21, 2010 tial guess of the parameter, with the possibility of slightly different initial value causing the algorithm to converge to an entirely different solution. One important parameter required by the algorithm is derivative of the function, which is not always available or may be difficult to calculate. In practical application, the recursive least square (RLS) technique is also employed to identify the parameters and fluxes of induction motor [3]-[4]. In [5]-[6], authors use the extended Kalman filter technique for the identification of the rotor time constant or a rotor resistance. However, this method presents some disadvantages, such as the influence of noise characteristics, computational burden, and deficiency of the design and tuning criteria. Other approaches, such as spectral analysis technique, have been applied to identify IM parameters [7]. The observed stimulus-response data in most of these studies have been used to identify the parameters, and the criterion of the response data is used as an objective function to be minimized. An objective function is a function of the squared predictive errors.

Recently, genetic algorithms (GA) have been used for motor parameter identification [8]-[9]. GA is a stochastic search technique borrowing concepts from biological evolutionary theory. Unlike other conventional techniques, GA enables the acquisition of the best set of parameter values by minimizing errors between the model evaluated and the plant. GA does not require derivative of the cost function. Therefore, the parameters to be estimated can cover all of the values in their research space. The genetic procedure consists in minimizing a cost function. The cost function is computed as a weighted sum either of square or absolute differences of the output variables, generally acquired experimentally, or those of the model computed by simulation.

However, in the papers cited above, the weights of the cost function are taken as constant values and not varying along the procedure of the algorithm. Since the cost function plays an important role in GA-based parameter identification, we propose an improved version of GA. The con- 
tribution of this work focuses on the influence of the variable weights of the cost function on the convergence speed to the global optimal parameter vector of the GA technique with a few generations. We use GAs for IM parameter identification, with the cost function given by the weighted sum of the stator current and the rotor speed errors.

The rest of this paper is organized as follows. Section 2 explains the GA procedure and the proposed fitness function. Section 3 gives the mathematical models of IM. Section 4 presents the parameter identification with the GA. Finally, Section 5 illustrates the obtained parameter identification results with the proposed approach.

\section{Genetic Algorithm}

\subsection{The Concept of Genetic Algorithm}

GAs are search algorithms, which use operations found in natural genetics to guide the trek through a search space. GAs use a direct analogy of natural behavior. They work with a population of chromosomes, each one representing a possible solution to a given problem. Each chromosome is assigned a fitness score according to how good a solution to the problem it is.

GAs are theoretically and empirically proven in order to provide robust search in complex spaces, giving a valid approach to problems requiring efficient and effective searching [10].

The concept of GAs, first formalized by Holland [11] and extended to functional optimization by Jong [12], involves the use of optimization search strategies patterned after Darwinian notion of natural selection and evolution. During a GA optimization, a set of trial solutions is chosen and "evolved" toward an optimal solution. Similar to nature, GAs find good chromosomes by blindly manipulating the material in the chromosomes without any knowledge about the type of problem they are solving. The only information they are given is an evaluation (or fitness) of each chromosome they produce.

GAs feature a group of candidate solutions (population) in the search space. Chromosomes with better fitness are found through natural selection and genetic operators, mutation, and recombination. Natural selection ensures that chromosomes with the best fitness will propagate in future populations. Using the recombination operator, the GA combines genes from two parent chromosomes to form two new chromosomes (children) with high probability of exhibiting better fitness than their parents would. Mutation allows new areas of the response surface to be explored. Given a problem, a way or method of encoding the solutions of the problem into the form of chromosomes must be determined. Second, an evaluation function that returns a measurement of the cost value (fitness) of any chromosome in the context of the problem should be defined.

Although there are many possible variants of a simple $\mathrm{GA}$, the fundamental underlying it operates on a population of chromosomes and consists of three operations:
- Evaluation of individual fitness;

- Formation of the gene pool (intermediate population); and

- Recombination and mutation.

The general framework of the procedure is described in Fig. 1 [13], where $\mathrm{P}(\mathrm{t})$ denotes the population at generation $\mathrm{t}$ :

begin

$\mathrm{t}:=0$

initialize the population $\mathrm{P}(\mathrm{t})$;

evaluate the population $\mathrm{P}(\mathrm{t})$;

while (not termination condition) do begin

$\mathrm{t}:=\mathrm{t}+1$;

select $\mathrm{P}(\mathrm{t})$ from $\mathrm{P}(\mathrm{t}-1)$;

alter $\mathrm{P}(\mathrm{t})$;

evaluate $\mathrm{P}(\mathrm{t})$;

end,

end.

Fig. 1. Procedure of genetic algorithms.

The advantages to using GAs are many: they require no knowledge or gradient information, they are resistant to becoming trapped in local optima, they perform very well for large-scale optimization problems, and they can be employed for a wide variety of optimization problems. However, they do have some disadvantages, and these include having trouble finding the exact global optimum and requiring a large number of fitness function evaluations or iterations. This is more obvious in situations when the dimensionality of the problem is large.

\subsection{Proposed Fitness Function}

The fitness function plays an important role to the GA optimization procedure as it measures the quality of the represented solution. The fitness function is always problem -dependent. An ideal fitness function closely connects with the algorithm's goal. Yet, it may be evaluated quickly because usually a genetic algorithm must be repeated many times to achieve the desirable result, and the speed of execution might be a very important factor.

To evaluate the cost function corresponding to an individual of the population, it is necessary to define the constant values of the weights that minimize the global cost. This function is given by

$$
O b j=\sum C_{i} f_{i}
$$

The choice of cost function is the key point of the GA procedure since this function is the indicator of the appropriateness of a potential solution vector, which could be computed using any domain knowledge.

To improve the overall performance and convergence speed of the GA procedure, a cost function that is the sum of the squared errors between the relevant points on gener- 
ated and modeled data is minimized.

With the aim of reducing the effort of calculus needed to evaluate the cost function in large search space, and hence, the speed convergence towards the optimal parameters of the plant, a simplified cost function will be regarded as the weighted sum of two squared error functions according to stator current and rotor speed of the IM. The weights will not have constant values along the identification procedure with the GA technique. The chromosomes include not only system parameters but also the weighing parameters of the cost function.

\section{Induction Motor Model}

Induction motor can be described by the following state equations in the synchronous d-q reference:

$$
\left\{\begin{array}{l}
\dot{x}_{1}=a_{11} x_{1}+a_{13} x_{3}+a_{14} x_{4} x_{5}+b_{1} v_{d s} \\
\dot{x}_{2}=a_{11} x_{2}-a_{14} x_{3} x_{5}+a_{13} x_{4}+b_{1} v_{q s} \\
\dot{x_{3}}=a_{31} x_{1}+a_{33} x_{3}-x_{4} x_{5} \\
\dot{x}_{4}=a_{31} x_{2}+x_{3} x_{5}+a_{33} x_{4} \\
\dot{x_{5}}=a_{51} x_{1} x_{4}-a_{51} x_{2} x_{3}-a_{52} T_{1}
\end{array}\right.
$$

where $\left[\begin{array}{lllll}x_{1} & x_{2} & x_{3} & x_{4} & x_{5}\end{array}\right]^{T}=\left[\begin{array}{lllll}i_{d s} & i_{q s} & \varphi_{d r} & \varphi_{q r} & w_{r}\end{array}\right]^{T}$

$i_{d s}, i_{q s}$ are the stator currents; $\varphi_{d r}, \varphi_{q r}$ are the rotor flux; $v_{d s}, v_{q s}$ are the stator voltages, $T_{1}$ is the load torque, and $w_{r}$ is the motor angular velocity.

$$
\begin{aligned}
& a_{11}=-\left(\frac{1}{\sigma L_{s}}\right)\left(R_{s}+\frac{L_{m}^{2}}{L_{r} \tau_{r}}\right) \\
& a_{13}=\frac{k}{\tau_{r}} \quad a_{14}=k \quad a_{31}=\frac{L_{m}}{\tau_{r}} \\
& a_{33}=-\frac{1}{\tau_{r}} \quad a_{51}=-\frac{3 P^{2} L_{m}}{8 J L_{r}} \quad a_{52}=\frac{P}{2 J} \quad b_{1}=\frac{1}{\sigma L_{s}} \\
& k=\frac{L_{m}}{\sigma L_{s} L_{r}} \quad \sigma=1-\left(\frac{L_{m}^{2}}{L_{s} L_{r}}\right) \quad \tau_{r}=\frac{L_{r}}{R_{r}}
\end{aligned}
$$

where $R_{s}$ and $R_{r}$ are the stator and rotor resistances, $L_{s}$ and $L_{r}$ are the stator and rotor inductances, $L_{m}$ is the mutual inductance, $\sigma$ is the leakage coefficient, $\tau_{r}$ is the rotor time constant, and $P$ and $J$ are the number of poles and inertia of the motor, respectively.

\section{Parameter Identification of IM using GA Procedure}

This section deals with parameter identification of IM described by the dynamic model (Eq. 2) with the GA pro- cedure. The parameter identification problem can be described as:

$$
\dot{X}=f(P, X, V)
$$

where $X$ : state variable vector;

$V$ : input vector from the supply; and

$P$ : parameter variable vector to be identified; and where

$$
P=\left[p_{1} p_{2} p_{3} \ldots p_{l}\right]^{T}
$$

The measurement vector is

$$
Y\left(t_{k}\right)=\left[y_{1}\left(t_{k}\right), y_{2}\left(t_{k}\right), \ldots y_{i}\left(t_{k}\right) \ldots, y_{m}\left(t_{k}\right)\right]
$$

where $t$ represents the values of the independent variable, $y_{i}$ is the state variable, and $m$ is the number of variables of the corresponding dependent variable to be measured.

With the given data, the optimal parameter vector, $\hat{P}$ can be identified:

$$
F(\hat{P})=\min _{P \in \square^{n}} F(P) ; \quad F(P)=\sum_{i=1}^{m}\left[y\left(t_{i} ; P\right)-y_{i}\right]^{2}
$$

In this paper, we shall consider the problem of determining the optimal parameters of the defined mathematical model using a set of differential equations defined in (2). State (2) is a nonlinear differential equation for the solution, whereby the fourth order Runge Kutta method is employed. Let

$$
\begin{aligned}
& X=\left[i_{d s}, i_{q s}, \varphi_{d r}, \varphi_{q r}\right]^{T} \\
& V=\left[v_{d s}, v_{q s}\right]^{T} \\
& P=\left[R_{s}, R_{r}, L_{s}, L_{r}, L_{m}, \ldots .\right]^{T}
\end{aligned}
$$

As described above, (2) can be interpreted as:

$$
\dot{X}=f(P, X, V)
$$

The measurement (generated) vector used in this study is

$$
Y\left(t_{k}\right)=\left[i_{d s}\left(t_{k}\right), i_{q s}\left(t_{k}\right), w_{r}\left(t_{k}\right)\right]^{T}
$$

The minimization problem may be solved by applying the GA. The parameter identification blocs procedure with the GA are shown in Fig.2.

Let $g$ be the sequence number of generations. The application of the GA to parameter identification can be described as follows: 
1. An initial population of parameters,

$$
\tilde{P}^{(0)}=\left\{\hat{P}_{i}^{(0)} \mid i=1,2, \ldots \text { popsize }\right\}
$$

is formed with randomly selected individuals. Each individual parameter vector is constrained by the following condition:

$$
p_{\min j} \leq \hat{p}_{i j}^{(g)} \leq p_{\max j} \quad j=1,2, \ldots, m
$$

where $p_{\min j}$ and $p_{\max j}$ are the limits of the $j$ th element of the parameter vector $\hat{p}_{i j}^{(0)}$ given by a prior knowledge. Symbol $\hat{p}_{i j}^{(g)}$ denotes the estimation of the $j$ th element of the $i t h$ individual in the $g$ th generation of the parameter. The process starts with $g=0$.

The initial ranges must be large enough to contain the global optimum. However, if made too large, the GA might not be able to find the global optimum at the required parameter identification accuracy within reasonable timeperiods; thus, the convergence will be slow and the efficiency poor. Any wildly inaccurate parameter may cause the machine model (an integral part of the identification procedure) to produce ridiculous results or generate run time errors.

2. Each individual $\hat{p}_{i}^{(g)}$ is used to calculate state variable $\hat{X}\left(t_{k}\right)$ and the general performance $\hat{Y}\left(t_{k}\right)$ corresponding to the measurement.

$$
\hat{\dot{X}}\left(t_{k}\right)=f\left(\hat{p}_{i}^{(g)}, \hat{X}\left(t_{k}\right), V\left(t_{k}\right)\right)
$$

The parameter optimization problem may be stated as follows: Determine the values of the ordered set of $\mathrm{n} \mathrm{pa-}$ rameters $P=\left[\begin{array}{llll}p_{1} & p_{2} & p_{3} & \ldots \\ p_{l}\end{array}\right]^{T}$, which minimize the cost function of the GA procedure.

The structure of the cost function is written as:

$$
\begin{aligned}
J=T_{s} C_{1} \sum_{k=1}^{N} & {\left[\frac{\left(i_{d s}\left(t_{k}\right)-\hat{i}_{d s}\left(t_{k}\right)\right)^{2}}{100}+\frac{\left(i_{q s}\left(t_{k}\right)-i_{q s}\left(t_{k}\right)\right)^{2}}{100}\right] } \\
& +T_{s} C_{2} \sum_{k=1}^{N} \frac{\left(w_{r}\left(t_{k}\right)-\hat{w}_{r}\left(t_{k}\right)\right)^{2}}{400}
\end{aligned}
$$

Squared current error and speed error are divided by 100 and 400 for normalization respectively, $\mathrm{N}$ represents the number of the signal point, and Ts denote the sampling time.

$\mathrm{C}_{1}$ and $\mathrm{C}_{2}$ are the weights to be optimized with GA. They fall in the interval [0 1], and they do not have to be the zero value in the same cost function.

3. A new generation $\hat{P}^{(g+1)}$, with the same individual number of $\hat{P}^{(g)}$, is formed by reproduction, crossover, and mutation based on the previous $\hat{P}^{(g)}$.

4. A process will stop if $g=\max g$, and $P=\hat{P}^{(g)}$ corresponds to the minimum of the cost function. Symbol $\max g$ is a big integer representing the maximum generation number; otherwise, $g=g+1$, and the process is repeated from Step 2.

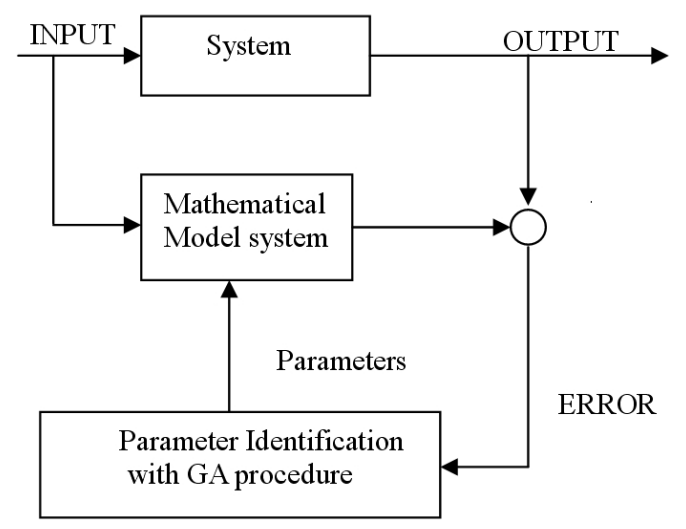

Fig. 2. GA parameter identification.

\section{Simulation Results}

The GA with an optimized cost function described in Section 2 can be employed to identify the parameters of the induction motor's dynamic model. This procedure is employed offline for parameter identification based on no load startup.

In this section, we give an overall specification of IM for data generation and parameter identification:

$$
\begin{aligned}
& f_{s}: \text { Fundamental frequency }=60 \mathrm{~Hz} \\
& V: \text { Line to line voltage }=220 \mathrm{~V} \\
& P: \text { Number of poles }=4 \\
& R_{s}=0.286 \Omega, R_{r}=0.246 \Omega, L_{S}=68.12 \mathrm{mH} \\
& L_{r}=70.33 \mathrm{mH}, L_{m}=66 \mathrm{mH}, J=0.3 \mathrm{Kg} \mathrm{m^{2 }} .
\end{aligned}
$$

Fig. 3 depicts the simulated stator current and rotor speed signals of an induction motor with no load.

We assume the procedure of GA is employed to identify the parameters in the dynamic model of IM under the condition of no load $(\operatorname{Tr}=0)$. The parameter vector to be identified by the GA with an optimized cost function in this study consists of the resistances and self-inductances of the stator and rotor winding $P=\left[R_{s} R_{r} L_{s} L_{r} L_{m} J\right]^{T}$. The state variables are stator current $I_{s}\left(t_{k}\right)=\left[i_{d s}\left(t_{k}\right), i_{q s}\left(t_{k}\right)\right]^{T}$ and rotor speed of the motor $w_{r}\left(t_{k}\right)$.

The methodology used to identify the IM parameters is 

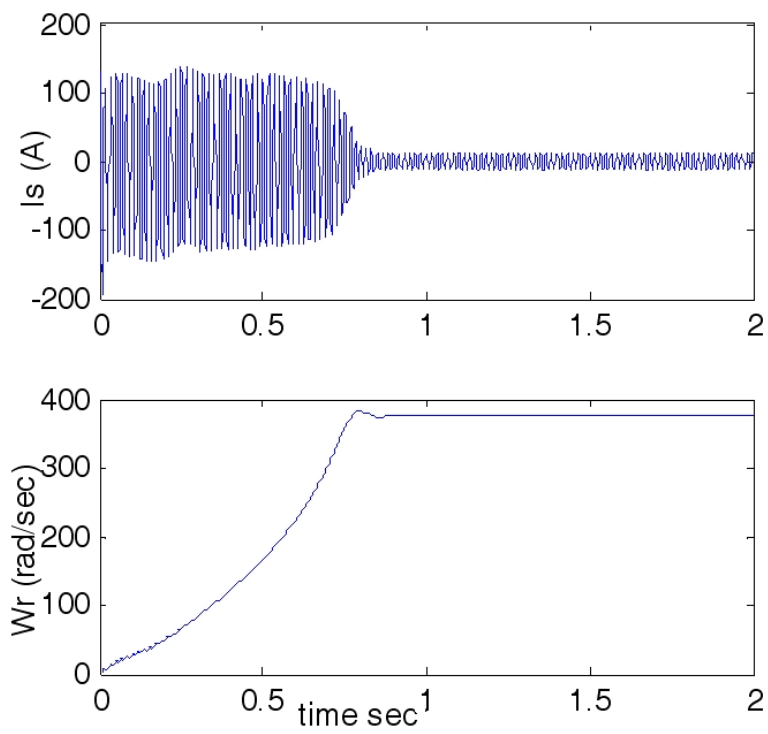

Fig. 3. Simulation of IM with no load.

illustrated in Fig. 4. The proposed method uses the data obtained from the simulation of a motor using the RungeKutta algorithm (this method could also be used with experimental data), where $I_{\mathrm{s}}, \mathrm{V}$, and $\mathrm{Wr}$ are the stator current, stator voltage, and rotor speed, respectively.

The test of the proposed identification procedure is performed only via simulation by emulating the real motor with a known model. In the identification process, the values of the parameters are supposed to be unknown and optimized.

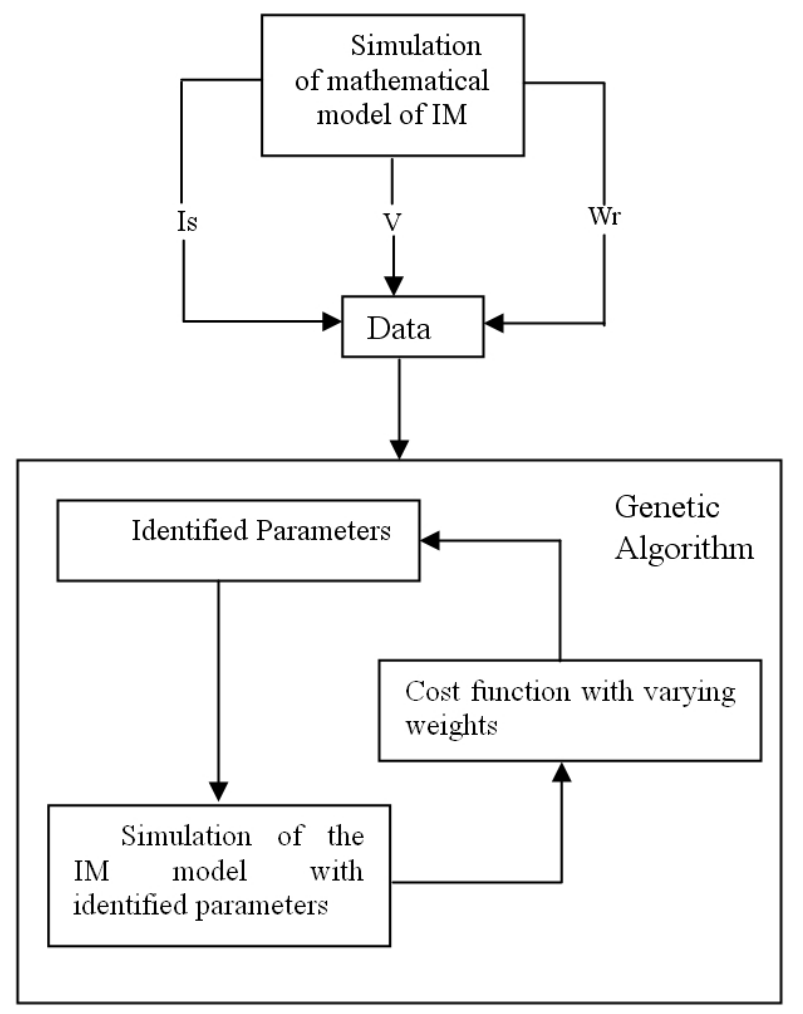

Fig. 4. Methodology of parameter identification.
Parameter identification is performed by the improved GA, which selects a set of parameter values at each iteration, and evaluates the response of the simulated motor. If the mismatch between experimental and simulated results below is at a fixed satisfactory level, we could say that the electrical parameters are identified; otherwise, the optimization procedure will continue until the target condition is verified.

The specifications of the GA used to identify the parameter vector $P$ used in this work are shown in Table 1 .

Table 1. Genetic Algorithm Characteristics

\begin{tabular}{c|c|c}
\hline Parameters & value & Description \\
\hline Popsize & 50 & Population size \\
\hline Length & 12 & Bit length for each parameter \\
\hline Maxgen & 100 & Maximum generation \\
\hline Pcross & 0.6 & Crossover rate \\
\hline $\mathrm{Pm}$ & 0.01 & Mutation rate \\
\hline
\end{tabular}

Along the optimization procedure with GA, generation was selected using binary tournament selection. For each slot in the new population, the better of two randomly selected individuals are copied. An elitist strategy retains the best individual.

The proposed GA is run with random initial population. The $C_{1}$ and $C_{2}$ are the weights optimized with GA in the interval [0 1].

Seven initial runs are performed to achieve this using a traditional GA with a dynamic value of weighting of the cost function. The obtained results are shown in Table II. The values of the fitness function $(F)$ are unitless quantities. Table 2 shows that best parameters identified are those displayed in the fourth row of the table.

The manufactured motor parameters used to generate the data, along with the best solution $\left(4^{\text {th }}\right.$ solution from Table 2) of identifying the problem for the IM model, are shown in Table 3.

Fig. 5a shows the results of the cost function with the optimized weights $\mathrm{C}_{1}$ and $\mathrm{C}_{2}$ (indicated in the fourth row of Table 2), where this cost function decreases with an increasing value of the generation number, and the gene of the chromosome almost converges near the 74th generation. The number of generations needed to reach a value of fitness under 0.05 is 42 .

Fig. 6 presents the percentage error (\%) of the identified parameters that are evaluated from the following expression:

$$
\operatorname{error}(\%)=\left|\frac{P-\hat{P}}{P}\right| \times 100
$$

where $\mathrm{P}$ denotes the identified parameter.

Fig. 7 shows the convergence of the GA to identify the parameters of IM. It also presents the best value of the lof subsequent parameters in each generation. The best values are obtained at the $73 \mathrm{rd}$ generation. 
Table 2. The Genetic Algorithm-Identified Motor Parameters With seven runs

\begin{tabular}{c|c|c|c|c|c|c|c|c|c}
\hline $\mathrm{N}$ & $\mathrm{C}_{1}$ & $\mathrm{C}_{2}$ & $\mathrm{Rr}$ & $\mathrm{Rs}$ & $\mathrm{Lr}$ & $\mathrm{Ls}$ & $\mathrm{L}_{\mathrm{m}}$ & $\mathrm{J}$ & $\mathrm{F}$ \\
\hline 1 & 0.013431 & 0.007814 & 0.249743 & 0.277838 & 0.070426 & 0.067868 & 0.067704 & 0.301076 & 0.010200 \\
\hline 2 & 0.131135 & 0.004395 & 0.244542 & 0.295494 & 0.068105 & 0.070311 & 0.065767 & 0.291897 & 0.000200 \\
\hline 3 & 0.094993 & 0.091330 & 0.245860 & 0.286813 & 0.070004 & 0.068399 & 0.065822 & 0.308725 & 0.000519 \\
\hline 4 & 0.013675 & 0.091575 & 0.246007 & 0.285970 & 0.069582 & 0.068824 & 0.066785 & 0.305865 & 0.000196 \\
\hline 5 & 0.013431 & 0.007814 & 0.249743 & 0.277838 & 0.070742 & 0.067761 & 0.065767 & 0.303941 & 0.000204 \\
\hline 6 & 0.094993 & 0.091330 & 0.245860 & 0.286813 & 0.069793 & 0.068611 & 0.065822 & 0.301655 & 0.000382 \\
\hline 7 & 0.227838 & 0.004395 & 0.250183 & 0.273663 & 0.069687 & 0.068717 & 0.066350 & 0.307980 & 0.000411 \\
\hline
\end{tabular}

Table 3. Results of Simulation Test with Improved GA

\begin{tabular}{c|c|c}
\hline Parameters & MANUFACTURED & Identified \\
\hline $\mathrm{Rr}$ & 0.2460 & 0.246007 \\
\hline $\mathrm{Rs}$ & 0.2877 & 0.285970 \\
\hline $\mathrm{Ls}$ & 0.06812 & 0.068824 \\
\hline $\mathrm{Lr}$ & 0.07033 & 0.069582 \\
\hline $\mathrm{L}_{\mathrm{m}}$ & 0.06600 & 0.066785 \\
\hline $\mathrm{J}$ & 0.300000 & 0.305865 \\
\hline
\end{tabular}

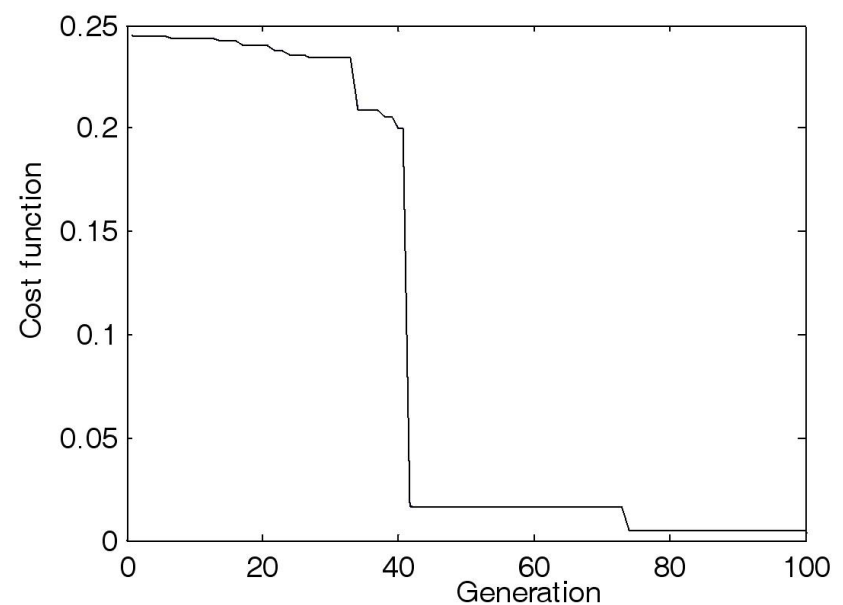

(a)

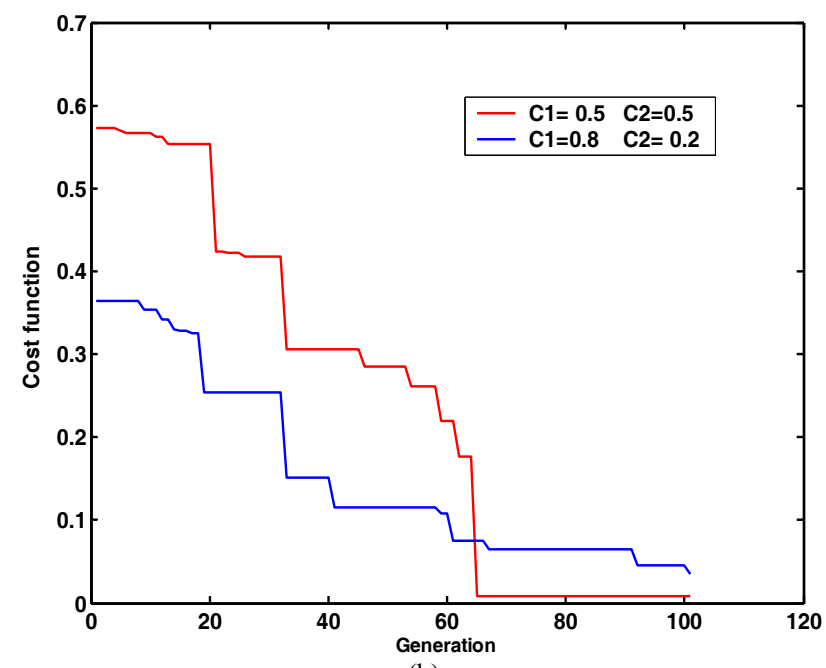

(b)

Fig. 5. The minimization of cost function:

(a) with variable weights and (b) constant weights.
The identified self-inductance error are all under $2.2630 \%$ at the $42 \mathrm{nd}$ generation and take constant values at the 65 th generation equal to $1.2130 \%$. The best values of the rotor and stator resistance are obtained at the 74 th generation.

The parameter values of the IM obtained with the modified GA proposed in this work are less than $5 \%$ of the real motor. Parameter identification at Grandfos Management $\mathrm{A} / \mathrm{S}$ [13] is considered highly satisfactory if the percent wise deviation is less than $5 \%$ from the true value, which is the best precision obtainable on the real motor using traditional system identification techniques.

These results show that the convergence is obtained in 74 generations with this proposed genetic algorithm
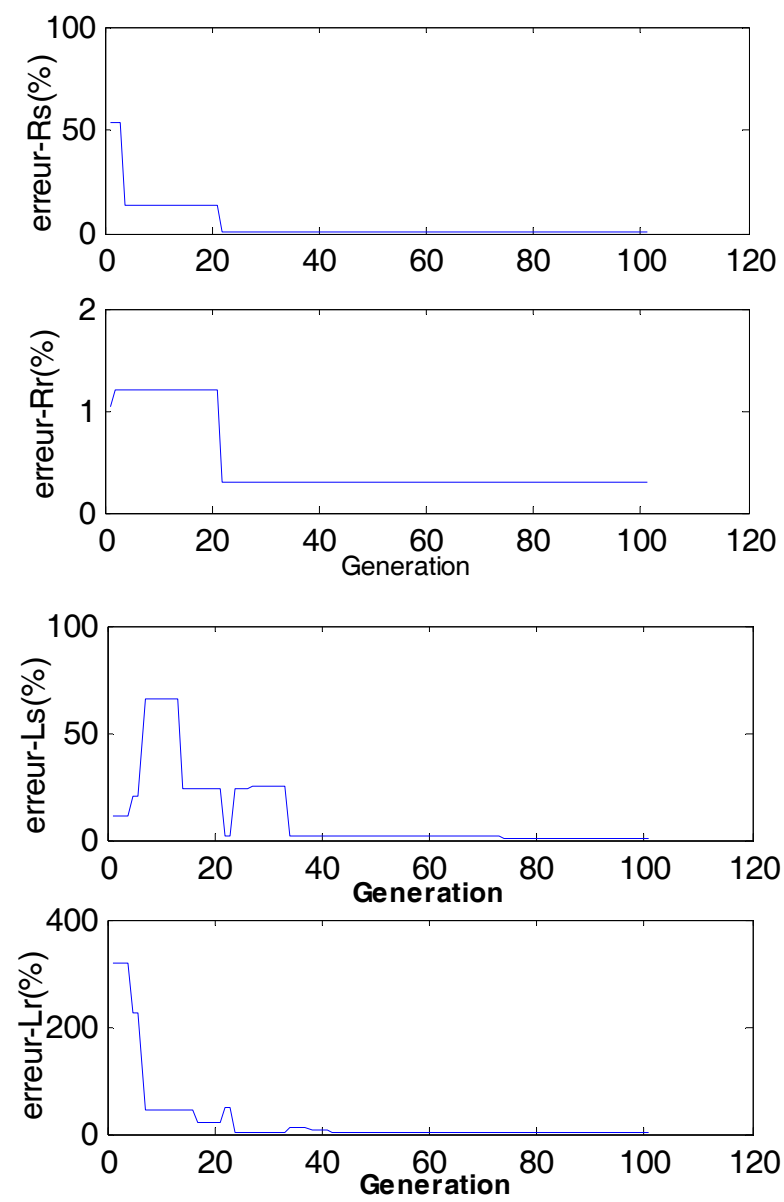

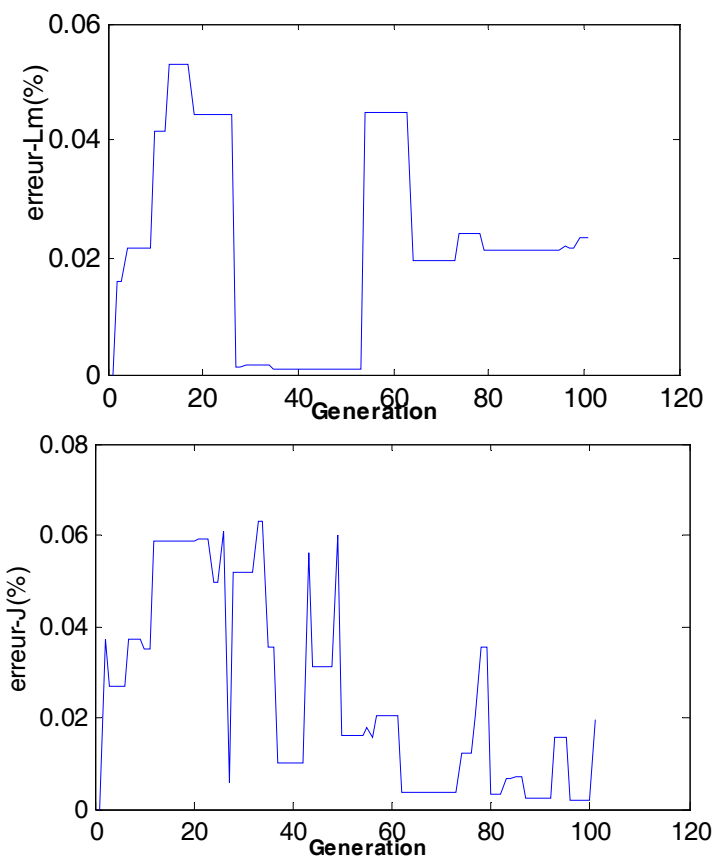

Fig. 6. Minimization of the IM parameters error (\%).
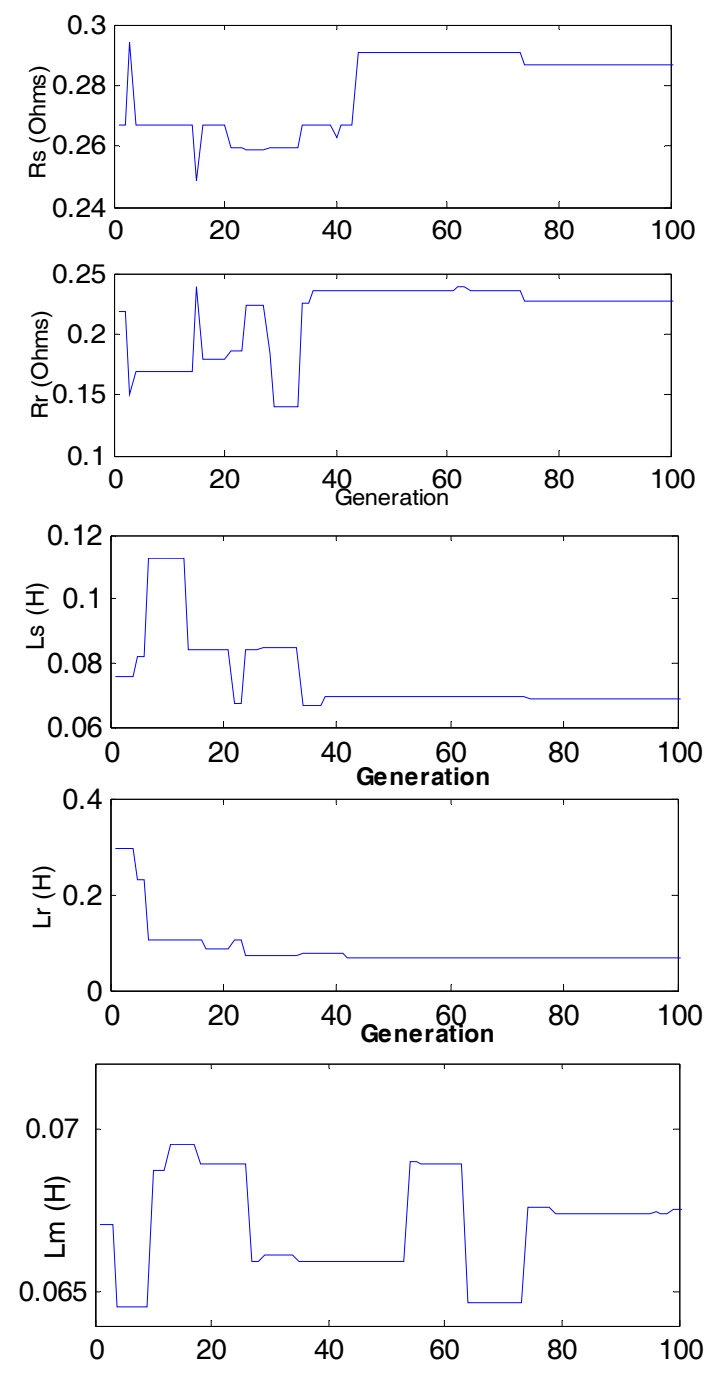

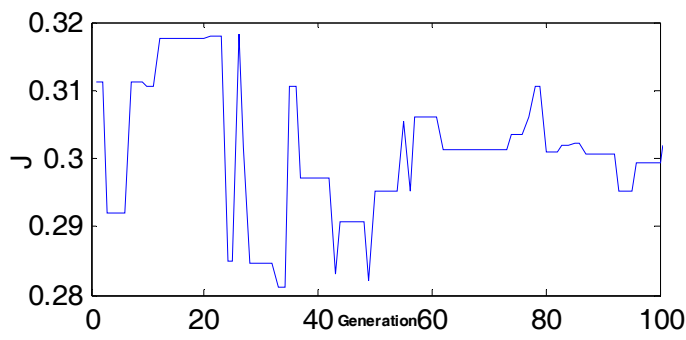

Fig. 7. General algorithm-identified parameters.

Comparison of the cost function with variable weights along with those with constant values of the GA in Fig. 5b shows excellent performance in terms of speed convergence and execution time given in Table 4, where the comparison of each algorithm is under the same condition of the characteristics of the GA in the same search space.

Table 4. Comparison of Execution Time of GA with Constant and Variable Weights of the Cost Function

\begin{tabular}{c|c|c|c}
\hline \multirow{2}{*}{ Cost Function } & $\begin{array}{c}\text { WITH } \\
\text { VARIABLE } \\
\text { WEIGHTS }\end{array}$ & $\begin{array}{c}\mathrm{C}_{1}=0.5 \\
\mathrm{C}_{2}=0.5\end{array}$ & $\begin{array}{l}\mathrm{C}_{1}=0.8 \\
\mathrm{C}_{2}=0.2\end{array}$ \\
\hline Execution time & $13.5 \mathrm{~min}$ & $20 \mathrm{~min}$ & $23 \mathrm{~min}$ \\
\hline
\end{tabular}

Regarding convergence speed, Figs. 5a and 5b show that the GA with variable weights of cost function is the fastest converging algorithm using the number of generation needed to reach a value of fitness under 0.05 as convergence speed measure. The GA with constant weights of cost function needed nearly more generations (more than 60 generations) to reach that fitness value.

A comparison of stator current response between the model of induction motor with manufactured value of parameters and the model using the new improved GA identified parameters in 0.1 second is shown in Fig. 8.

The system dynamic behavior obtained using the proposed GA parameter identification perfectly matches that of the model with the real ones.

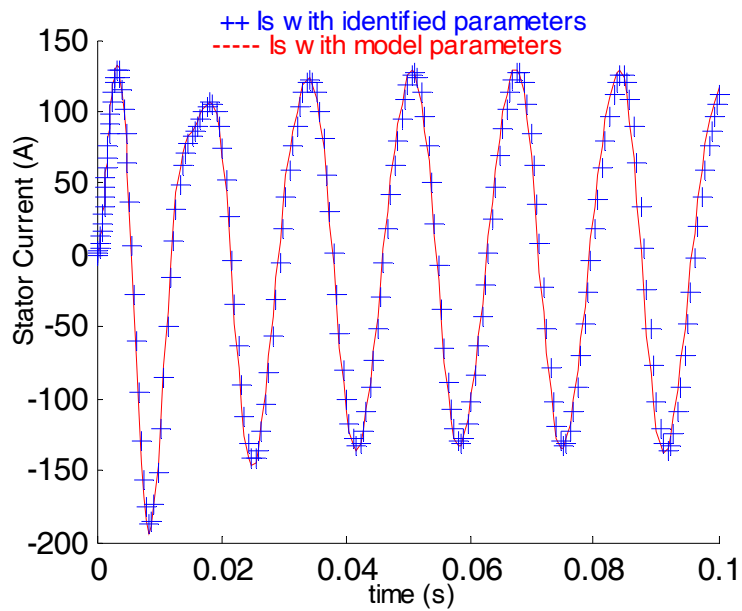

Fig. 8. Stator current obtained with identified IM parameters and the true model parameters. 


\section{Conclusion}

The main objective of this study is to identify the parameters of IM. The GA proposed showcases good overall converging feature, giving solution to the problem of traditional identification methods.

GAs with optimized weights of cost function can identify the IM model parameters with less error. The errors for all parameters evaluated from (12) are 5\% lower than the a priori set tolerance.

The optimized weights of the cost function influence the speed convergence of the GA procedure and the final identified results. From the simulation, GA finds that the identified parameters are almost the same as those of the real system. Despite the good identification capability of GA, it also has a limitation. Its considerably long execution time confines its application only to off line identification.

\section{Acknowledgements}

This work was supported by the MESRS Algeria under the research project CNEPRU, J0201420070042.

The authors would like to thank Professors A. Hamzaoui and N. Essounbouli from Crestic Laboratory, IUT Troyes France for their help in achieving the objective of this study.

\section{References}

[1] P. Vas, Sensorless vector and direct torque control: Oxford University Press, 1998.

[2] Tahir Sağ and Mehmet Çunkaş, "Multiobjective Genetic Estimation to Induction Motor Parameters", in Proceedings of ACEMP'07 \& electromotion'07, international Conference, Bodrum, Turkey, September 2007.

[3] J. Stephan, M. Bodson, and J. Chiasson, "Real-time estimation of the parameters and fluxes of induction motors", IEEE Trans. Ind. Applicat, Vol. 30, No.1, pp. 746-759, June 1994.

[4] Y. Koubaa, "Asynchronous machine parameters estimation using recursive method", Simulation Modelling Practice and Theory, Elsevier Vol. 14, pp 10101021, 2006

[5] L.-C. Zai, C. L. De Marco, and T. A. Lipo, "An extended Kalman filter approach to rotor time constant measurement in PWM induction motor drives", IEEE Trans. Ind. Applicat., Vol. 28,. pp. 96-104, February 1992.

[6] M. Menaa, O. Touhami, R. Ibtiouen, "Estimation of the rotor resistance in induction motor by application of the spiral vector theory associate to extended Kalman filter", IECON conference of the IEEE, Vol. 1, 2003.

[7] H. Chai and P. Acarnley, "Induction motor parameter estimation algorithm using spectral analysis", Proc.
Inst Elect. Eng., Elect. Powe Applicat., Vol. 139, No. 3, pp. 165-174, May 1992.

[8] F. Alonge, F. D'lppolito, G. Ferrante, and F. M. Raimondi, "Parameter identification of induction Motor model using genetic algorithms", Proc.Inst. Elect. Eng., contr. Theory Applicat., Vol. 145, N. 6, pp. 587-593, November 1998.

[9] F. Alonge, F. D'lppolito, G. Ferrante, and F. M. Raimondi, "Least squares and genetic algorithms for $\mathrm{Pa}-$ rameter identification of induction motor", Control engineering practice, Elsevier, 2001, pp. 647-657.

[10] D. E. Goldberg, "Genetic Algorithms in search optimization, and Machine Learning", Addison Wesley. Reading, MA, 1989.

[11] J. H. Holland, "Adaptation in natural and artificial system" The University of Michigan Press, Ann Arbor, 1975.

[12] D. Jong, "Analysis of the behavior of a class of genetic adaptive systems". Ph.D. dissertation, The University of Michigan Press, 1975.

[13] Z. Michalewicz, Genetic Algorithms + Data Structures =Evolution Programs, Springer-extended edition, Berlin, 1996.

[14] R. K. Ursem and P. Vadstrup, "Parameter identification of Induction motors using stochastic optimization algorithms", Applied soft computing journal, Vol. 4, pp. 49-64. Elsevier 2004.

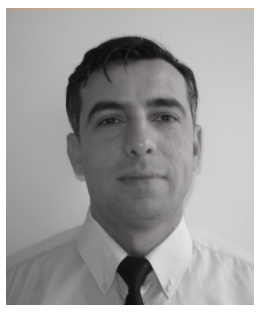

Ahmed Chaouki Megherbi received his Electrical Engineer and Magister grades from the University of Biskra and Batna University respectively, (Algeria), in 1993 and 2000 . He is currently working on his $\mathrm{PhD}$ thesis. From 2000-2010, he was with the Electrical Engineering Institute of Bechar University. At present, he is with Biskra University. His research interests are control and system identification based on soft computing methods.

Hassina Megherbi received her BSc in Electronics in 1995 from the Department of Electronics Engineering from the University of Med Khider Biskra, Algeria. She also received an MSc degree in Electronics in 1998 from the University of Ferhat Abass Setif (Algeria). She is currently assigned at the Automatic Department. Her research is focused on the robot control using the fuzzy neuronal network and evolutionary algorithm. She is a member of LSI (Laboratoire des systèmes Intelligents) University of Ferhat Abbas Setif. 
Khier Benmahammed received his MSc degree in Electronics in 1980 from University of Colorado, USA, and $\mathrm{PhD}$ degree in Computer Science in 1986 from University of Louisiana, USA. He is IEEE Senior Member. He is currently a Professor of Electronics Engineering. His current research interests are image processing, fuzzy system control, and clustering.

\begin{abstract}
Abdel Ghani Aissaoui was born in 1969 in Moghrar, Naama, Algeria. He received his BS degree in electrical engineering from the Electrical Engineering Institute of University of Sidi Bel Abbes (Algeria) in 1993, his MS degree from the Electrical Engineering Institute of University of Sidi Bel Abbes in 1997 and his PhD from the Electrical Engineering Institute of University of Sidi Bel Abbes in 2007.

He is currently Professor of Electrical Engineering at University of Bechar (Algeria). He is a member of IRECOM (Interaction Réseaux Electrique - Convertisseurs Machines) Laboratory. His current research interest includes power electronics and control of electrical machines.
\end{abstract}

Ahmed Tahour was born in 1972 in Ouled Mimoun, Tlemcen, Algeria. He received his BS degree in Electrical Engineering from the Electrical Engineering Institute of University of Sidi Bel Abbes in 1996, and his MS degree from the Electrical Engineering Institute of University of Sidi Belabbes in 1999 and his Ph.D. from the Electrical Engineering Institute of the University of Sidi Bel Abbes in 2007. He is currently Professor of Electrical Engineering at University of Bechar (Algeria). His current research interests include power electronics and control of electrical machines. 